\title{
A Simple Equation for the Energy Stored by Voltage- Dependent Capacitances
}

\author{
Utkarsh Jadli, Student Member, IEEE, Faisal Mohd-Yasin, Senior Member, IEEE, Hamid Amini Moghadam, Jordan \\ R. Nicholls, Peyush Pande, and Sima Dimitrijev, Senior Member, IEEE
}

\begin{abstract}
The parasitic capacitances of semiconductor power devices that contribute to the switching losses are voltagedependent, which can make calculations of their stored energy difficult. Typically, manufacturers will provide effective capacitance values to aid in circuit design and component selection. However, stored energy calculations using these effective capacitor values are erroneous. In this paper, we derive a new equation for the stored energy in the voltage-dependent capacitance associated with a semiconductor depletion region, such as in diodes and transistors. In particular, we show that the $1 / 2$ term in $1 / 2 C V^{2}$ should be replaced by a new term $\gamma$, which depends on the device structure. By applying our proposed method to several commercial diodes and transistors, we show that it matches the measured data much better than using the effective capacitances. The proposed equation will enable better power circuit design by improving the accuracy of stored energy calculations. ${ }^{1}$
\end{abstract}

Index Terms - Coss losses, effective capacitance, energy stored, Eoss, switching losses, simple equation, voltage dependent parasitic capacitances.

\section{INTRODUCTION}

$I^{r}$ NCREASE in switching frequencies have driven modern power converters towards greater power densities and smaller converter sizes. However, with the increase in the switching frequencies, the switching characteristics-and crucially the switching losses - of the power semiconductor devices become highly impactful and may compromise the overall power efficiency. The switching characteristics of the power semiconductor devices are highly dependent on the voltage-dependent parasitic capacitances. At high frequencies, the effect of these voltage dependent capacitances is profound, but their impact is largely overlooked by the researchers [1]-[4].

The output capacitance $\left(C_{O S S}\right)$ of a power semiconductor device is the primary voltage-dependent capacitance of interest as it actively participates in the switching transitions. For a hard switched converter, Coss is charged during turn-Off interval, and then discharges the same stored energy through the channel of the power semiconductor device during turn-On interval [5]-[7]. Hence, it plays a crucial role in the distribution of switching losses. In soft switching circuits, Coss plays a significant role in determining the resonant frequency for

This work was supported by Innovative Manufacturing Cooperative Research Centre (IMCRC), and BlueGlass as the industry partner for project no: 220132.

The authors are with the Queensland Micro- and Nanotechnology Centre, Griffith University, Queensland, 4111, Australia, and the School of creating zero-voltage switching (ZVS) conditions [3]. Therefore, $C_{O S S}$ cannot be ignored for power circuits. A greater insight into $C_{O S S}$, and in particular the impact of its voltage dependencies, will be useful for designing more efficient power converters.

Generally, Coss versus applied drain-to-source voltage curves are provided by the manufactures in their datasheets, which is useful for circuit analysis. Modern datasheets also provide energy stored in the COSS with increasing drain-tosource voltage $\left(v_{D S}\right)$. The CoSS stored energy can also be computed by integrating $C_{O S S}\left(v_{D S}\right) \cdot v_{D S}$ product from zero to a chosen $V_{D S}$ value [8]. Additionally, the manufacturers also provide effective capacitances in their datasheets. The primary reason for this is to translate the voltage-dependent capacitances into voltage-independent values of the effective capacitances, so that traditional linear methods can be applied to analyze the power circuits [7], [9].

The effective capacitance that is widely used for calculating the energy stored in the $C_{O S S}\left(v_{D S}\right)$ is defined as the fixed output capacitance that will store the same amount of energy as $C_{O S S}\left(v_{D S}\right)$ does when $v_{D S}$ rises from $0 \mathrm{~V}$ to $80 \%$ of the maximum drain-to-source voltage $\left(V_{D S}=0.8 V_{D S-M A X}\right)$ [7], [10]. According to this definition, the value of the effective capacitance, which we will label by $C_{e f f}$, is calculated by the following equation [11]:

$$
\frac{1}{2} V_{D S}{ }^{2} C_{e f f}=\int_{0}^{V_{D S}} C_{O S S}\left(v_{D S}\right) \times v_{D S} d v_{D S}
$$

Approximating the voltage-dependent capacitance of power semiconductor devices with the voltage-independent effective capacitance $\left(C_{e f f}\right)$ simplifies circuit analysis and calculations of the dissipated energy. However, it can also result in significant errors [7]. The concept of $C_{\text {eff }}$ is correct for the specific $V_{D S}$ point selected as the upper integral boundary in (1), meaning that the energy calculated using $1 / 2 C_{\text {eff }} V_{D S}{ }^{2}$ is correct only for a single specified $V_{D S}$ point and gives wrong results for all other $v_{D S}$ voltages.

This paper addresses the problem in calculating energy stored using $C_{\text {eff. }}$. For the case of constant capacitances, $E=1 / 2 C V^{2}$ is valid, but this simple equation does not apply for the case of voltage-dependent capacitors. In this paper, we show

Engineering and Built Environment, Griffith University, Queensland, 4111, Australia (email: utkarsh.jadli@griffithuni.edu.au; f.mohdyasin@griffith.edu.au; $\quad$ h.aminimoghadam@griffith.edu.au; jordan.nicholls@griffithuni.edu.au; peyush.pande@griffithuni.edu.au; s.dimitrijev@griffith.edu.au). 


\section{IEEE POWER ELECTRONICS REGULAR PAPER/LETTER/CORRESPONDENCE}

that the factor $1 / 2$ in the constant capacitance equation can be modified and used with the actual measured capacitance of the power semiconductor device to obtain correct values of stored energy $\left(E_{O S S}\right)$ at any voltage across the capacitor. Our approach provides a simple equation that can fit the stored energy in the voltage-dependent capacitances of diodes, power MOSFETs, superjunction (SJ) MOSFETs, and HEMTs.

To demonstrate the theoretical foundation of this approach, which is to modify the $1 / 2$ term rather than the voltage-dependent capacitance, we derive the equation for energy stored by the parasitic capacitance in semiconductor diodes in the next section.

\section{DERIVATION OF THE FUNDAMENTAL EQUATION FOR ENERGY STORED IN PARASITIC CAPACITANCES OF SEMICONDUCTOR DIODES}

\section{A. Linear $P-N$ Junction}

The depletion-layer width $(W)$ of linear $\mathrm{P}-\mathrm{N}$ junction is given by [12]:

$$
W=\left(\frac{12 \varepsilon_{s}}{a} \varphi\right)^{1 / 3}
$$

where, $\mathcal{E}_{s}$ is the semiconductor permittivity, $\varphi$ is the electric potential, and we have expressed the linear change of doping concentration by $N_{D}=a x$. Note that the depletion layer in the $\mathrm{N}$-type region is equal to $W / 2$. The charge stored in the N-type region (the cathode) of this capacitor is given as [12]:

$$
Q=A(a / 8) W^{2}
$$

where, $A$ is the area of the linear $\mathrm{P}-\mathrm{N}$ junction. Differentiating (3) and (2), we obtain

$$
\begin{gathered}
d Q=A(a / 4) W d W \\
d W=\frac{1}{3}\left(\frac{12 \varepsilon_{s}}{a}\right)^{1 / 3} \varphi^{-2 / 3} d \varphi
\end{gathered}
$$

The differential capacitance of the depletion layer for linear P$\mathrm{N}$ junction can now be calculated using (2), (4), and (5):

$$
C_{d}=\frac{d Q}{d \varphi}=A\left(\frac{a}{12}\right)^{1 / 3} \varepsilon_{s}^{2 / 3} \varphi^{-1 / 3}=\beta \varphi^{-1 / 3}
$$

where $\beta=A(a / 12)^{1 / 3} \varepsilon_{s}^{2 / 3}$.

In the case of a constant capacitance, $C$, the well-known equation for the stored energy is obtained in the following way:

$$
E=\int_{0}^{V} \varphi d Q=\int_{0}^{V} \varphi C d \varphi=\frac{1}{2} C V^{2}
$$

However, in the case of a voltage-dependent capacitor, (7) has to be modified. The voltage-dependent capacitance has to be integrated together with the electric potential, and the boundaries of the integral need to account for the relationship between the applied voltage and the electric potential across the depletion layer of the diode. Using (6) for the case of a linear $\mathrm{P}-\mathrm{N}$ junction, we have

$$
E=\int_{V_{b i}}^{V+V_{b i}} \varphi C_{d} d \varphi=\frac{3}{5} \beta\left[\left(V+V_{b i}\right)^{5 / 3}-V_{b i}^{5 / 3}\right]
$$

In terms of $C_{d}=\beta\left(V+V_{b i}\right)^{-1 / 3}$, (8) can be rewritten as:

$$
E=\frac{3}{5} C_{d}\left(V+V_{b i}\right)^{2}-\frac{3}{5} C_{d}\left(V+V_{b i}\right)^{1 / 3} V_{b i}^{5 / 3}
$$

For high reverse bias voltages, $V_{b i}<<V$, and $V_{b i}$ can be ignored in (9). Therefore, the energy stored in the voltage-dependent capacitance of a linear $\mathrm{P}-\mathrm{N}$ junction can be approximated by:

$$
E \approx \frac{3}{5} C_{d} V^{2}
$$

The comparison between (10) and (7) shows the difference between the stored energy in a voltage-dependent capacitor and in a fixed capacitor.

\section{B. One-Sided Abrupt P-N Junction and Schottky Diodes}

The derivation for one-sided abrupt $\mathrm{P}-\mathrm{N}$ junctions and Schottky diodes, which is the opposite extreme case from the linear $\mathrm{P}-\mathrm{N}$ junction, is analogous [12]. The following is the result for the energy stored in one-sided abrupt $\mathrm{P}-\mathrm{N}$ junctions and Schottky diodes:

$$
E \approx \frac{2}{3} C_{d} V^{2}
$$

Again, the comparison between (11) and (7) shows the difference between the stored energy in a voltage-dependent capacitance and in a fixed capacitor.

\section{PROPOSED METHOD}

The equation for the depletion-layer capacitance of an arbitrary $\mathrm{P}-\mathrm{N}$ junction, which is widely used by the researchers for modelling the voltage-dependent capacitance of power semiconductor devices, is given by [5], [7], [12]:

$$
C_{d}(V)=C_{d}(0)\left[1+\left(V / V_{b i}\right)\right]^{-m}
$$

Here, $C_{d}(0)$ is the zero bias capacitance and $m$ is the grading coefficient. Note that the grading coefficient $m$ is equal to $1 / 2$ for the case of an abrupt P-N junction, $1 / 3$ for the case of a linear P$\mathrm{N}$ junction, and it can take a value between $1 / 2$ and $1 / 3$ for the case of an arbitrary doping profile at the $\mathrm{P}-\mathrm{N}$ junction. For high voltages, $V / V_{b i} \gg 1$, and (12) can be rewritten as:

$$
C_{d}(V)=C_{d}(0) V_{b i}^{m} V^{-m}
$$

The energy stored in this voltage dependent capacitance is:

$$
\begin{gathered}
E=\int_{0}^{V} C_{d}(V) V d V=\int_{0}^{V} C_{d}(0) V_{b i}{ }^{m} V^{-m} V d V \\
E=\frac{C_{d}(0) V_{b i}^{m} V^{-m}}{2-m} V^{2}=\frac{C_{d}(V) V^{2}}{2-m}
\end{gathered}
$$

To simplify (15), we introduce the term $\gamma=1 /(2-m)$, which leads to the final form of the proposed equation:

$$
E=\gamma C_{d}(V) V^{2}
$$

Note that (16) becomes $E=1 / 2 C V^{2}$ for $m=0$, which is the energy stored in fixed capacitance, exactly like (7). 


\section{IEEE POWER ELECTRONICS REGULAR PAPER/LETTER/CORRESPONDENCE}

Furthermore, (16) represents the energy stored in both the abrupt and linear $\mathrm{P}-\mathrm{N}$ junctions, as derived in Section II, with $\gamma=2 / 3$ and $\gamma=3 / 5$, respectively (the two extreme cases described in the previous section). For an arbitrary doping profile at the $\mathrm{P}-\mathrm{N}$ junction, $\gamma$ values could be between $3 / 5$ and $2 / 3$. Therefore, we propose (16) to be used as a simple equation for calculating the energy stored in voltage-dependent capacitances, where $\gamma$ is a variable parameter that can be used to fit different doping profiles.

The next section will show the implementation of (16) in commercial diodes.

\section{IMPLEMENTATION IN COMMERCIAL DIODES}

As mentioned in the previous section, the doping profile of real junctions lies between the abrupt and liner extremes. Therefore, the energy stored in the parasitic capacitances of a real diode will be between the extreme values given by (10) and (11), which means that $3 / 5<\gamma<2 / 3$. The actual value of this parameter for a specific diode can be determined by fitting (16) to the dependence of stored energy on voltage, obtained by $\int C_{d}(V) V d V$. Note that by proposing (16) we enable device manufacturers to provide the value of $\gamma$ in their datasheets, instead of either the simple but incorrect $C_{\text {eff }}$ or the graphs of stored energy versus voltage that are not suitable for analyses by analytical equations.

To verify the proposed approach, we applied it to three commercial diodes: STPS10L25D, RFNL20TJ6S, and SB5100. Capacitance-voltage $(C-V)$ measurements for the respective diodes were performed with an Agilent Power Device Analyzer (B1505A) using four-point probe measurement. The proposed method was applied to the aforementioned diodes and the results are shown in Fig. 1. Note that $C_{\text {eff }}$ for each of the diodes was calculated using (1) and the respective $C_{\text {eff }}$ values are: $761 \mathrm{pF}$ for STPS10L25D, $107.9 \mathrm{pF}$ for RFNL20TJ6S, and $92.4 \mathrm{pF}$ for SB5100.

The two simple equations, the usual $(1 / 2) C_{e f f} V^{2}$ and the newly proposed $\gamma_{d}(V) V^{2}$, are compared to the correct value of the stored energy, $\int C_{d}(V) V d V$, which was calculated from the measured $C_{d}-V$ data. It is clear from Fig. 1 that a significant error is being made when using $C_{\text {eff. }}$. On the other hand, the proposed equation (16) fits very closely to the correct values of the stored energy for all diodes and at all values of applied voltage. It can be also noticed that the $\gamma$ values for all the diodes are in the derived theoretical range for diodes with doping profiles between the abrupt and linear extremes.

\section{APPLICATIONS TO POWER MOSFETS, SUPERJUNCTION MOSFETS, AND HEMTS}

While our proposed method has been derived for the case of diodes, we can also apply it to the output capacitances of power MOSFETs, and HEMTs. This is because these parasitic capacitances are primarily caused by depletion regions in the device structure and the voltage dependences are quite similar to those of diodes.

Furthermore, the proposed equation can be applied to superjunction (SJ) MOSFETs with a slight modification to
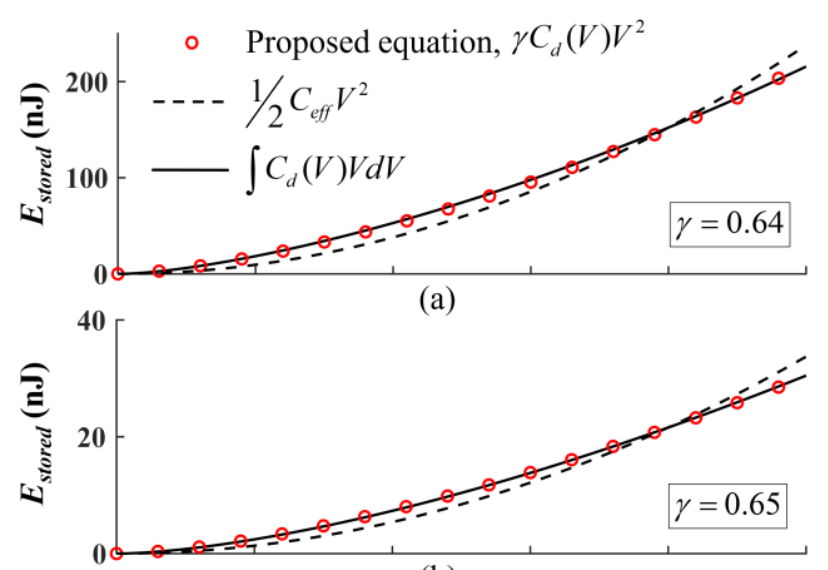

(b)

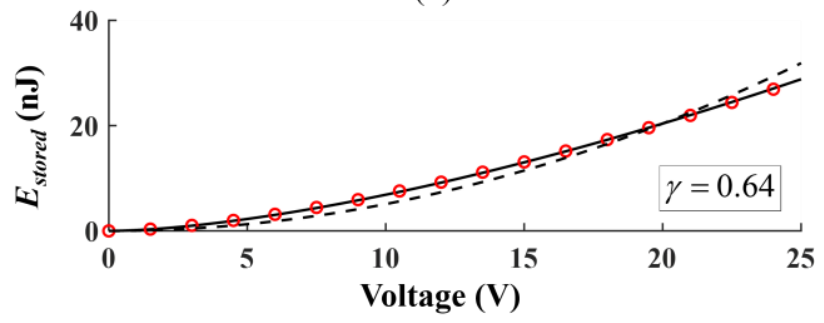

(c)

Fig. 1. Energy stored in the parasitic capacitance of (a) STPS10L25 (b) RFNL20TJ6S (c) SB5100 using different methods.

account for the complex structure of this device. The typical capacitance-voltage dependence of these devices consists of two distinct ranges [13]. The first is a low-voltage range, which is mainly determined by the 3-D surface of the blocking $\mathrm{P}-\mathrm{N}$ junctions. However, the N-type region between the P-type columns is fully depleted at relatively low voltages (typically, less than $10 \%$ of the blocking voltage). When this occurs, any further voltage increase beyond this boundary voltage increases the depletion layer in the N-type drift region. This is the second range, where the equation we have derived for the drift region in diodes is applicable. Since this high-voltage range begins at voltages smaller than $10 \%$ of the blocking voltage, we can conclude that the specific $E(V)$ dependence in the low voltage range is of no interest. However, the energy stored at the boundary voltage when the device enters the high-voltage range has to be added to the $E(V)$ equation for the high-voltage range. Therefore, we propose a modification in the derived equation by adding a constant-energy term, $E_{C O N S T}$, to account for the energy stored at the boundary voltage: $E=\gamma_{d}(V) V^{2}+E_{C O N S T}$.

A 300-V Si power MOSFET (IRFB4137PbF) from Infineon, a 100-V enhancement-mode GaN HEMT (GS61004B) from GaN Systems, a 650-V SJ MOSFET (IPB65R045C7) from Infineon, and a 650-V SJ MOSFET (FCHD125N65S3R0) from ON Semiconductor were selected to test the proposed approach. The manufacturers have provided the $E_{O S S}$ plot for the respective devices, which will be used to verify the proposed equation. We used the grabit.m command in the MATLAB to extract the $C_{O S S}$ and $E_{O S S}$ data from the respective datasheets. For better resolution, data points between those taken from the datasheet were generated via interpolation. $C_{\text {eff }}$ values quoted in the datasheet for IRFB4137PbF, GS61004B, IPB65R045C7, 


\section{IEEE POWER ELECTRONICS REGULAR PAPER/LETTER/CORRESPONDENCE}
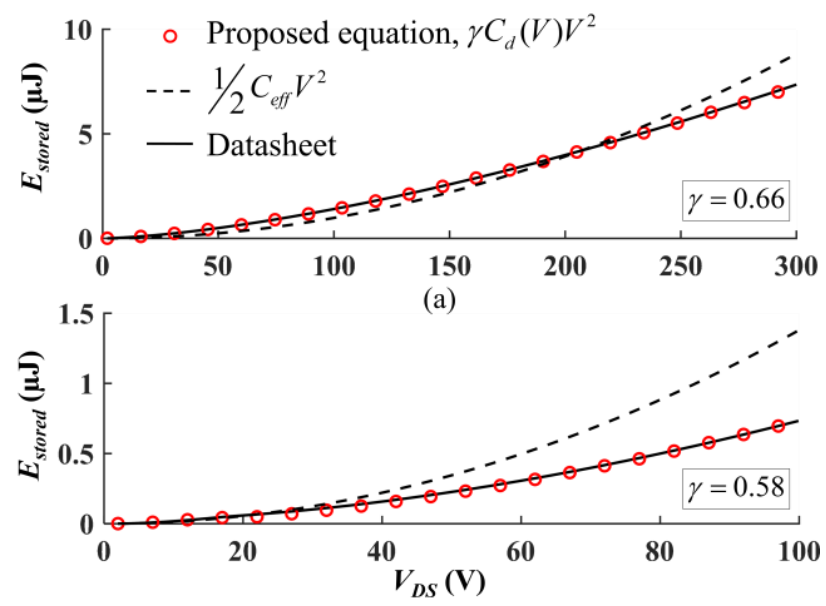

(b)

Fig. 2. Energy stored in $C_{\text {osS }}$ of (a) Power MOSFET IRFB4137PbF and (b) GaN-HEMT GS61004B.

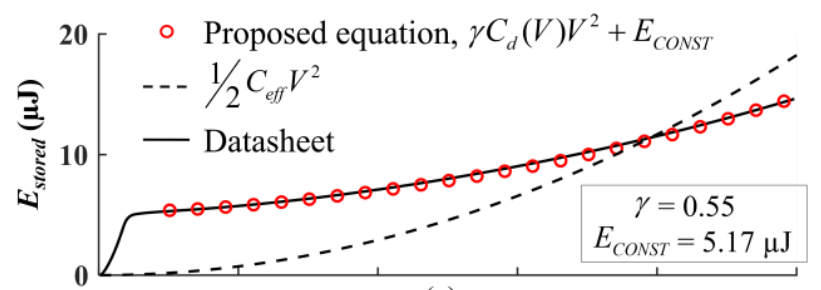

(a)

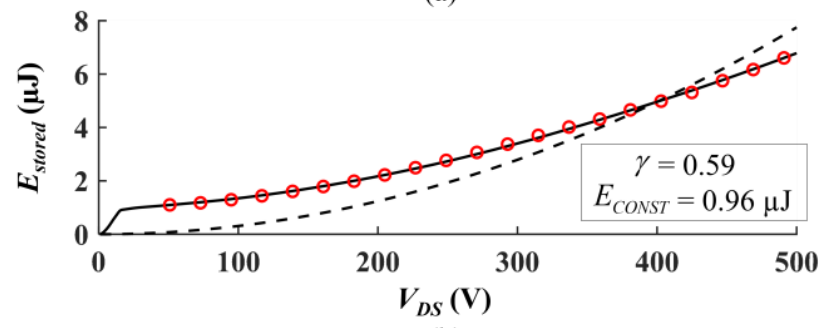

(b)

Fig. 3. Energy stored in $C_{\text {OSS }}$ of (a) SJ MOSFET IPB65R045C7 and (b) SJ MOSFET FCHD125N65S3R0.

and FCHD125N65S3R0 are equal to $196 \mathrm{pF}, 276 \mathrm{pF}, 146 \mathrm{pF}$, and $62 \mathrm{pF}$, respectively.

Unlike the diodes in the previous section, we allowed $\gamma$ for these selected devices to be unbounded. The proposed approach was applied to these selected devices and the corresponding energy stored in the $C_{O S S}$ is shown in Fig. 2 and Fig. 3. The values of $\gamma$ for IRFB4137PbF, GS61004B, IPB65R045C7, and FCHD125N65S3R0 obtained by fitting (16) to the energy values from the datasheets are $0.66,0.58,0.55$, and 0.59 , respectively. The values of $E_{\text {CONST }}$ for IPB65R045C7 and for FCHD125N65S3R0 are $5.17 \mu \mathrm{J}$ and $0.96 \mu \mathrm{J}$, respectively. It can be clearly seen that the proposed simple equation (16), with the modification for SJ MOSFETs, can match the datasheet values of $E_{\text {OSS. }}$ As distinct from this, the energy calculation using $C_{\text {eff }}$ only matches the energy stored at $V_{D S}=240 \mathrm{~V}$ for IRFB4137PbF, at $V_{D S}=400 \mathrm{~V}$ for the two SJ MOSFETs, and at smaller voltages for GS61004B. Importantly, the approach based on $C_{\text {eff }}$ cannot represent the entire voltage range. This clearly shows the error in energy calculations by the simple equation with constant capacitance $C_{\text {eff. }}$. Therefore the energy calculation from our proposed equation, as compared to using $C_{e f f}$, is much more accurate.

\section{CONCLUSION}

In this paper, we have proposed a replacement of the equation $E=1 / 2 C_{\text {eff }} V^{2}$ for the energy stored in the parasitic capacitances of diodes and power switches. We have demonstrated both theoretically and experimentally that the equation $E=\gamma C_{d}(V) V^{2}$, where $\gamma$ is a variable parameter and $C_{d}(V)$ is the actual measured capacitance, provides an excellent fit to the actual dependencies of stored energy on applied voltages for the case of commercial diodes, power MOSFETs, and HEMTs. For the case of superjunction MOSFETs, we have added a constant term, $E_{\text {CONST, }}$, to enable its application to the high-voltage range of interest for circuit design and analysis. The proposed equation will be useful for power circuit design by enabling fast, simple, and accurate calculations of the switching losses.

\section{REFERENCES}

[1] G. Zulauf, Z. Tong, J. D. Plummer and J. M. Rivas-Davila, "Active Power Device Selection in High- and Very-High-Frequency Power Converters," IEEE Trans. Power Electron., vol. 34, no. 7, pp. 6818-6833, July 2019.

[2] G. Zulauf, W. Liang, K. Surakitbovorn and J. Rivas-Davila, "Output capacitance losses in $600 \mathrm{~V} \mathrm{GaN}$ power semiconductors with large voltage swings at high- and very-high-frequencies," 2017 IEEE 5th Workshop on Wide Bandgap Power Devices and Applications (WiPDA), Albuquerque, NM, 2017, pp. 352-359.

[3] G. Zulauf, S. Park, W. Liang, K. N. Surakitbovorn and J. Rivas-Davila, "COSS Losses in $600 \mathrm{~V}$ GaN Power Semiconductors in Soft-Switched, High- and Very-High-Frequency Power Converters," IEEE Trans. Power Electron., vol. 33, no. 12, pp. 10748-10763, Dec. 2018.

[4] J. Wang, H. S.-h. Chung, and R. T.-h. Li, "Characterization and Experimental Assessment of the Effects of Parasitic Elements on the MOSFET Switching Performance," IEEE Trans. on Power Electron., vol. 28, no. 1, pp. 573-590, Jan. 2013.

[5] R. W. Erickson, "Switch Realization," in Fundamentals of Power Electronics, New York, NY, USA: Chapman \& Hall, 1997, pp. 94-104.

[6] Y. Xiong, S. Sun, H. Jia, P. Shea, and Z. J. Shen, "New physical insights on power MOSFET switching losses," IEEE Trans. Power Electron., vol. 24, no.2, pp. 525-531, Feb. 2009.

[7] D. Costinett, D. Maksimovic, and R. Zane, "Circuit-Oriented Treatment of Nonlinear Capacitances in Switched-Mode Power Supplies," IEEE Trans. on Power Electron., vol. 30, no. 2, pp. 985-995, Feb. 2015.

[8] Z. Tong, G. Zulauf, J. Xu, J. D. Plummer and J. Rivas-Davila, "Output Capacitance Loss Characterization of Silicon Carbide Schottky Diodes," IEEE Journal of Emerging and Selected Topics in Power Electronics, vol. 7, no. 2, pp. 865-878, June 2019.

[9] G. D. Zulauf, J. Roig-Guitart, J. D. Plummer and J. M. Rivas-Davila, "COSS Measurements for Superjunction MOSFETs: Limitations and Opportunities," IEEE Trans. on Electron Devices, vol. 66, no. 1, pp. 578584, Jan. 2019.

[10] International Rectifier Application Note An-1001 [Online]. Available: https://www.infineon.com/dgdl/an-1001.pdf?fileId=5546d462533600a4 $01535590 \mathrm{a} 5 \mathrm{c} 70 \mathrm{f} 36$

[11] Toshiba Power MOSFET Electrical Characteristics Application Note [Online]. Available: https://toshiba.semiconstorage.com/info/docget.jsp?did=13415

[12] S. Dimitrijev, Principles of Semiconductor Devices, $2^{\text {nd }}$ Ed., New York, Oxford University Press, 2012, pp. 312-316.

[13] F. Udrea, G. Deboy and T. Fujihira, "Superjunction Power Devices, History, Development, and Future Prospects," IEEE Trans. on Electron Devices, vol. 64, no. 3, pp. 713-727, Mar. 2017. 